\title{
Accurate measurement of scattering and absorption loss in microphotonic devices
}

\author{
Matthew Borselli, ${ }^{1,2}$ Thomas J. Johnson, ${ }^{1}$ and Oskar Painter ${ }^{1, *}$ \\ ${ }^{1}$ Department of Applied Physics, California Institute of Technology, Pasadena, California 91125, USA \\ ${ }^{2}$ Present address, Xponent Photonics Inc., Monrovia, California 91016, USA \\ *Corresponding author: opainter@caltech.edu
}

Received July 10, 2007; revised September 10, 2007; accepted September 10, 2007; posted September 12, 2007 (Doc. ID 85012); published October 5, 2007

We present a simple measurement and analysis technique to determine the fraction of optical loss due to both radiation (scattering) and linear absorption in microphotonic components. The method is generally applicable to optical materials in which both nonlinear and linear absorption are present and requires only limited knowledge of absolute optical power levels, material parameters, and the structure geometry. The technique is applied to high-quality-factor $\left(Q=1 \times 10^{6}\right.$ to $\left.Q=5 \times 10^{6}\right)$ silicon-on-insulator (SOI) microdisk resonators. It is determined that linear absorption can account for more than half of the total optical loss in the high- $Q$ regime of these devices. (C) 2007 Optical Society of America

OCIS codes: $230.3990,140.3945$.

The push for dense integration of photonic elements into existing microelectronics circuits has revitalized the interest in semiconductor microphotonics [1-4]. Unfortunately, the benefits of tight optical confinement provided by high-index-contrast photonic elements have oftentimes been offset by increased optical losses due to high modal overlap with imperfect surfaces damaged by processing or imperfectly defined by lithography. As advances in the etching and definition of these structures have reduced geometrical nonidealities, absorption has become a significant source of optical loss [5]. Understanding the optical losses of these structures is important for continued progress in developing low-loss microphotonic circuits; as a result, many recent articles [5-9] have detailed methods for inferring the amount of optical loss due to absorption.

Here we present a simple method for determining the linear absorption optical loss in microphotonic components without resorting to models of the thermal response of the structure or the character of the absorption. The devices studied in this work consist of high- $Q$ Si microdisk resonators formed from SOI wafers. As described in detail below, by monitoring the resonance wavelength and on-resonance transmission value of the microdisk modes as a function of input power, the relative amounts of linear absorption and radiation (scattering) loss within the resonator can be ascertained. While this implementation of the method is specific to the Si material system in which two-photon absorption (TPA) [10] is present, more generally it can be used in situations where there is some form of additional nonlinear absorption. The technique is also applicable to a wide variety of resonator geometries and thus can be employed for the study of optical loss in waveguides through the use of waveguide-based microring resonators.

The Si microdisks studied in this work consist of $10 \mu \mathrm{m}$ radius disks formed in a $217 \mathrm{~nm}$ thick Si device layer on top of a $2 \mu \mathrm{m} \mathrm{SiO}_{2}$ buried oxide layer. Details of the disk resonator fabrication process can be found in [5]. Device characterization was per- formed by using a tunable external-cavity laser $(\lambda$ $1420-1498 \mathrm{~nm}$, linewidth $<300 \mathrm{KHz}$ for time scales relevant to this work) connected to a computercontrolled fiber taper waveguide probe [6] and two optical attenuators as in Fig. 1(a). The $\mu \mathrm{m}$-scale fiber taper probe was formed from a standard single-mode optical fiber and used to evanescently excite the whispering-gallery modes (WGMs) of the microdisk with controllable loading. The two (highly linear) op-
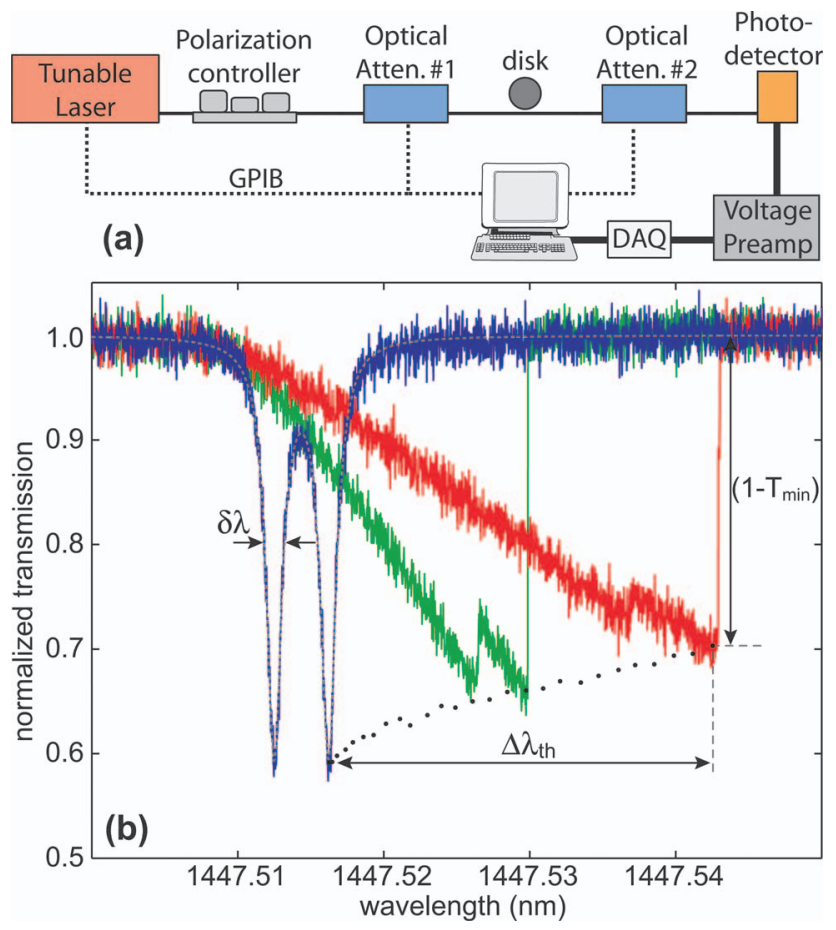

Fig. 1. (a) Schematic representation of testing apparatus. GPIB, general purpose interface bus; DAQ, data acquisition. (b) Measured transmission scans at various input powers for a $10 \mu \mathrm{m}$ radius Si microdisk. The input powers are 4,50 , and $100 \mu \mathrm{W}$ for the blue, green, and red curves, respectively. The fit to the low-power curve yields $\gamma_{t} / 2 \pi$ $=1.4 \times 10^{8} \mathrm{~Hz}, \quad \gamma_{\beta} / 2 \pi=4.2 \times 10^{8} \mathrm{~Hz}, \quad$ and $\quad \gamma_{e} / 2 \pi=3.3$ $\times 10^{7} \mathrm{~Hz}$. 
tical attenuators were controlled to provide variable optical input power to the resonators while maintaining a constant signal at the photodetector to eliminate nonlinearities in the detection electronics.

Figure 1(b) shows the normalized spectral transmission response of a $10 \mu \mathrm{m}$ radius microdisk for several different input powers. As is evident for the higher input powers, the thermo-optic effect of Si [11] leads to an optical bistability in the transmission scan. The observed doublet resonance dip, most discernible in the low-power (blue) curve, is a result of surface roughness coupling the normally degenerate clockwise (CW) and counterclockwise (CCW) propagating WGMs [12]. The resulting coupled modes can be conveniently described as sinelike and cosinelike standing wave modes with their respective modefield amplitudes $\left(a_{s}\right.$ and $a_{c}$ ) given by

$$
a_{c(s)}=\frac{-\sqrt{\gamma_{e} / 2} \sqrt{P_{i}}}{-\left(\gamma_{t} / 2\right)+i\left(\Delta \omega \pm \gamma_{\beta} / 2\right)},
$$

where $\Delta \omega$ is the detuning of the input laser frequency from the initially degenerate mode frequency $\omega_{0}, \gamma_{t}$ is the total decay rate of each individual resonance, $\gamma_{e}$ is the coupling rate into the fiber taper waveguide, $\gamma_{\beta}$ is the rate of CW to CCW mode coupling, $P_{i}$ is the optical input power at the fiber-cavity junction, and we take the upper sign for $a_{c}$ [6]. The normalized transmission of the resonator-waveguide system is

$$
T=\frac{\left|-\sqrt{P_{i}}+\sqrt{\gamma_{e} / 2}\left(a_{c}+a_{s}\right)\right|^{2}}{P_{i}} .
$$

Low-power transmission data [blue curve in Fig. 1(b)] is fitted to Eq. (2) and used to infer the resonator's total linear loss $\left(Q_{t} \equiv \omega_{0} / \gamma_{t} \approx \lambda_{0} / \delta \lambda=1.5 \times 10^{6}\right)$ and the other parameters of Eq. (1). The resulting fit is displayed as the orange dashed curve in Fig. 1(b). For clarity of presentation, the analysis presented below is for singlet resonances: the analysis for doublet resonances is conceptually the same.

To facilitate the treatment below, we separate the total optical loss rate into a cold-cavity portion $\left(\gamma_{c}\right)$ and a nonlinear absorption portion $\left(\gamma_{n l a}\right), \gamma_{t}=\gamma_{c}$ $+\gamma_{n l a}$. The cold-cavity loss rate comprises linear absorption $\left(\gamma_{l a}\right)$, radiation, and scattering loss. Similarly, the absorption coefficient $\left(\gamma_{a}\right)$ can be separated into linear and nonlinear contributions according to $\gamma_{a}=\gamma_{l a}+\gamma_{n l a}$. With these definitions of loss rates, for the steady-state absorbed power within the cavity we can write $P_{\mathrm{abs}}=\left(\gamma_{a} / \gamma_{t}\right) P_{d}$, where the dropped power $\left(P_{d}\right)$ is related to the input power and normalized resonant transmission minimum $\left(T_{\min }\right)$ through $P_{d}$ $=\left(1-T_{\min }\right) P_{i}$.

Following [13] we define the coupling factor, $K$, to be $\left(\gamma_{e} / \gamma_{t}\right)$. Recalling our partitioning of the total loss rate, we find $\gamma_{n l a}^{\prime}=\left(K_{0} / K\right)-1$, where $\gamma_{n l a}^{\prime}$ is given by $\left(\gamma_{n l a} / \gamma_{c}\right)$ and $K_{0}$ is taken to be the coupling factor at low power in the absence of nonlinear absorption, $K_{0}=\left(\gamma_{e} / \gamma_{c}\right)$. Relating the coupling factor to the onresonance transmission minimum yields

$$
\gamma_{n l a}^{\prime}\left(P_{d}\right)=K_{0}\left(\frac{1 \mp \sqrt{\left(T_{\min }\left(P_{d}\right)\right)}}{1 \pm \sqrt{\left(T_{\min }\left(P_{d}\right)\right)}}\right)-1,
$$

where the upper sign is taken in the undercoupled regime. Thus we can infer the normalized nonlinear absorption rate from the cold-cavity measurement of the coupling factor and the observation of minimum transmission depth versus dropped power. Figure 2 shows a plot of the inferred normalized nonlinear absorption for each of the measured transmission minima (dots) versus input power in Fig. 1(b). It should be noted that the absolute dropped power need not be known to find this quantity. The relative dropped power can be converted into a relative cavity energy $\left(U_{c}=P_{d} / \gamma_{t}\right)$ to observe the energy dependence of the normalized nonlinear absorption, as is done in Fig. 2. The linear dependence of $\gamma_{n l a}^{\prime}$ versus $U_{c}$ indicates that two-photon absorption (TPA) is the dominant nonlinear absorption inside the Si microdisks at these input powers (peak input power of $\sim 100 \mu \mathrm{W}$ ).

The remaining quantity of interest, $\gamma_{a}$, can be determined by considering the relationship between the resonance position and absorbed power due to the thermo-optic effect of Si. For small temperature changes we expect the resonance wavelength shift, $\Delta \lambda$, to be linearly proportional to the absorbed power [11]. Therefore, we have $\Delta \lambda=C\left(\gamma_{a} / \gamma_{t}\right) P_{d}$, where $C$ is a constant depending upon the thermal and thermooptic characteristics of the microdisks. Keeping in mind the decomposition of $\gamma_{a}$ and $\gamma_{t}$ above, we find

$$
\Delta \lambda\left(P_{d}\right)=C\left(\frac{\gamma_{l a}^{\prime}+\gamma_{n l a}^{\prime}\left(P_{d}\right)}{1+\gamma_{n l a}^{\prime}\left(P_{d}\right)}\right) P_{d},
$$

where $\gamma_{l a}^{\prime}$ is given by $\left(\gamma_{l a} / \gamma_{c}\right)$. With $\gamma_{n l a}^{\prime}\left(P_{d}\right)$ already measured from the resonant transmission versus input power, Eq. (4) indicates that $\gamma_{a}^{\prime}$ may be determined directly from the global slope curve, $\left[\Delta \lambda_{t h} / P_{d}\right]$ $\times\left(P_{d}\right)$. As $\gamma_{a}^{\prime}$ can be estimated from this slope curve alone, we need not know the absolute dropped power. Similarly, knowledge of the temperature change of

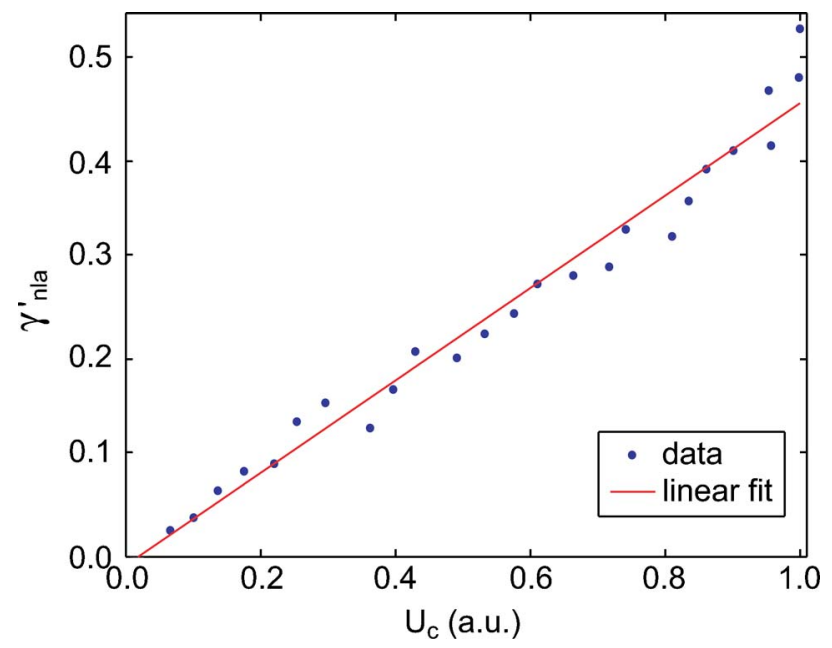

Fig. 2. (Color online) Plot of normalized nonlinear absorption versus relative electric field cavity energy along with a linear fit. 
the resonator and the thermal resistance of the structure, needed for directly estimating the absorbed power, is also not needed.

Figure 3(a) shows the measured resonance shift from Fig. 1(b) versus $P_{d}$ along with a fit to Eq. (4) and a linear fit to the lowest power data as a visual aid. The inset also plots the global slope curve, $\left[\Delta \lambda_{t h} / P_{d}\right]$ $\times\left(P_{d}\right)$, for the same data set. From a fit to the global slope curve we find that $\gamma_{l a}^{\prime}=0.57 \pm 0.03$ for this resonant mode. Analysis of the entire family of WGMs (varying azimuthal mode number, fixed radial order and polarization) for the same microdisk is plotted in Fig. 3(b). Linear absorption is found to persist at a nearly constant level of $\delta \lambda_{a}=0.5 \mathrm{pm}$ across the $1400 \mathrm{~nm}$ wavelength band, corresponding to a loss per unit length of roughly $0.25 \mathrm{~dB} / \mathrm{cm}$. As bulk absorption in the moderately $\mathrm{p}$-doped $\mathrm{Si}$ of these devices is expected to be more than an order of magnitude smaller [14] than this measured value, the linear absorption loss is attributed to the etched and polished surfaces of the disk. This is consistent with
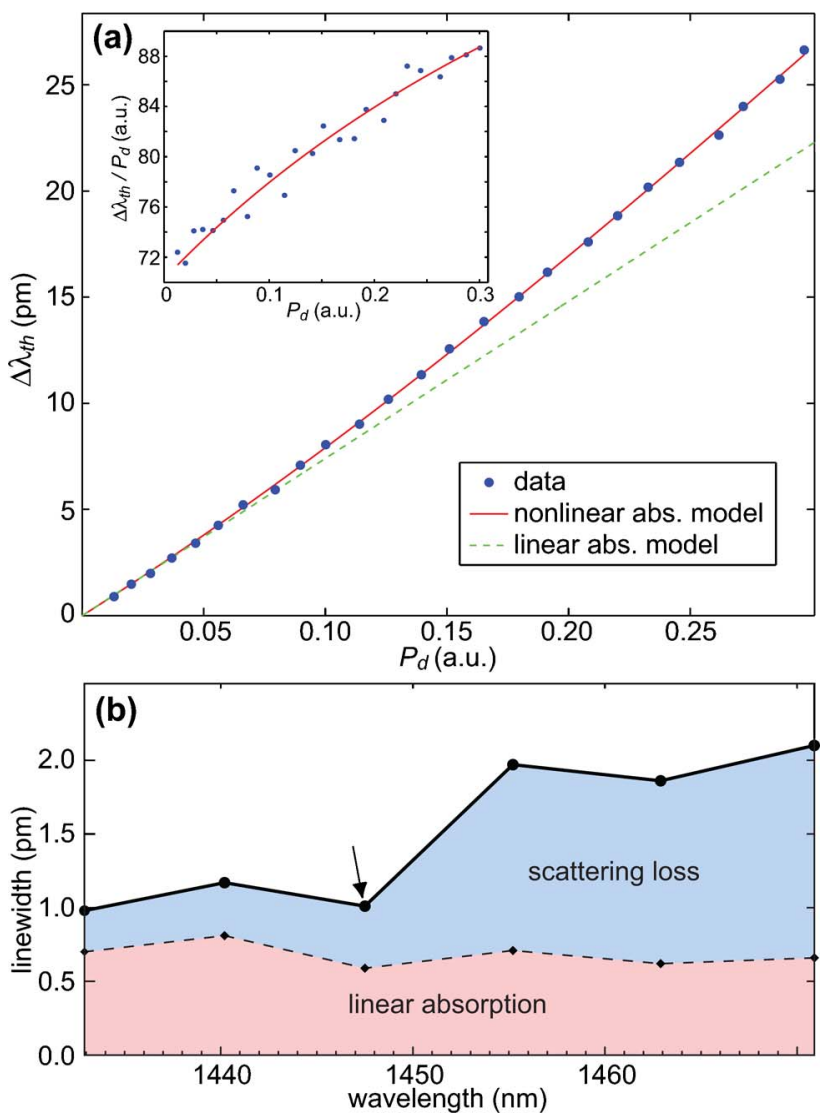

Fig. 3. (Color online) (a) Plot of thermally induced wavelength shift $\left(\Delta \lambda_{t h}\right)$ versus relative dropped power $\left(P_{d}\right)$ along with nonlinear and linear absorption model fits. Inset, global slope, $\Delta \lambda_{t h} / P_{d}$, versus $P_{d}$ for the same dataset. (b) Measured intrinsic linewidth for the entire family of high- $Q$ WGMs of this microdisk, along with the measured delineation between scattering loss and linear absorption. Intrinsic radiation loss of the WGMs is lumped in with the scattering loss component; however, it is calculated to be negligible for the $10 \mu \mathrm{m}$ radius disks studied here. The resonant mode of panel (a) is denoted with an arrow in this plot. recent studies we performed on similar devices in which optical absorption loss could be dramatically varied with changing chemistry of the Si surfaces [5]. A gradual trend of increasing scattering loss with wavelength can also be seen in Fig. 3(b). This is likely due to the smaller azimuthal wave vector of longerwavelength WGMs, which results in a reduced scattering momentum required for coupling into radiation modes.

As the above example serves to illustrate, for materials in which there is a measureable amount of two-photon absorption, by monitoring the resonance wavelength and transmission of a microresonator one can separate the amount of linear absorption from that of other loss components such as intrinsic radiation and scattering. More generally, this technique can provide insights into the nature of optical loss in other materials and devices utilizing nonlinear absorption to accurately determine linear absorption with only limited knowledge of absolute power, material parameters, and physical geometry of the structure.

This work was supported by the Defence Advanced Research Projects Agency through the Electronic Photonic Integrated Chip (EPIC) program. The authors thank Chris Michael, Paul Barclay and Kartik Srinivasan for useful discussions. M. B. Borselli thanks the Moore Foundation, National Physical Science Consortium, and HRL Laboratories for his graduate fellowship support.

\section{References}

1. S. J. Choi, K. D. Djrodjev, Z. Peng, Q. Yang, S. J. Choi, and P. D. Dapkus, IEEE Photon. Technol. Lett. 17, 2101 (2004).

2. Q. Xu, B. Schmidt, S. Pradhan, and M. Lipson, Nature 435, 325 (2005).

3. H. Rong, A. Liu, R. Jones, O. Cohen, D. Hak, R. Nicolaescu, A. Fang, and M. Paniccia, Nature 433, 292 (2005).

4. A. W. Fang, H. Park, O. Cohen, R. Jones, M. J. Paniccia, and J. E. Bowers, Opt. Express 14, 9203 (2006).

5. M. Borselli, T. J. Johnson, and O. Painter, Appl. Phys. Lett. 88, 131114 (2006).

6. M. Borselli, T. J. Johnson, and O. Painter, Opt. Express 13, 1515 (2005).

7. C. A. Barrios, V. R. Almeida, R. R. Panepucci, B. S. Schmidt, and M. Lipson, IEEE Photon. Technol. Lett. 16, 506 (2004).

8. I. Alvarado-Rodriguez and E. Yablonovitch, J. Appl. Phys. 92, 6399 (2002).

9. C. P. Michael, K. Srinivasan, T. J. Johnson, O. Painter, K. H. Lee, K. Hennessy, H. Kim, and E. Hu, Appl. Phys. Lett. 90, 051108 (2007).

10. M. Dinu, F. Quochi, and H. Garcia, Appl. Phys. Lett. 82, 2954 (2003).

11. G. Cocorullo and I. Rendina, Electron. Lett. 28, 83 (1992).

12. M. Gorodetsky, A. Pryamikov, and V. Ilchenko, J. Opt. Soc. Am. B 17, 1051 (2000).

13. P. E. Barclay, K. Srinivasan, and O. Painter, Opt. Express 13, 801 (2005).

14. R. A. Soref and B. R. Bennett, IEEE J. Quantum Electron. 23, 123 (1987). 